\title{
Three-dimensional visualization of flowing droplets and their analysis using real-time quantitative phase microscopy
}

Received 00th January 20xx, Accepted 00th January 20xx DOI: $10.1039 / \times 0 \times x 00000 x$

\author{
Yingdong Luo ${ }^{a}$, Jinwu Yang ${ }^{a}$, Xinqi Zheng ${ }^{a}$, Jianjun Wang ${ }^{a}$, Xin $\mathrm{Tu}^{\mathrm{a}}$, Zhizhao $\mathrm{Che}^{\mathrm{b}}$, Jiakun Fang ${ }^{\mathrm{c}}$, Lei \\ $\mathrm{Xi}^{\mathrm{d}}$, Nam-Trung Nguyen ${ }^{\mathrm{e}}$ and Chaolong Song *a
}

Recent years have witnessed the development of droplet-based microfluidics as an useful and effective tool for high-throughput analysis in biological, chemical and enviromental sciences. Despite the flourishing development of droplet manipulation techniques, few methods allow for label-free and quantitative inspection of flowing droplets in microchannels in real-time and in three dimensions (3-D). In this work, we propose and demonstrate the application of a real-time quantitative phase microscopy technique for 3-D visualization of droplets, and also for full-field and label-free measurement of analyte concentration distribution in the droplets. The phase imaging system consists of a linear-CCD-based holographic microscopy configuration and an optofluidic phaseshifting element, which can be used for retrieving quantitative phase maps of flowing objects in microchannels with a temporal resolution only limited to the frame rate of the CCD camera. To demonstrate the capabilities of the proposed imaging technique, we have experimentally validated the 3-D image reconstruction of the droplets generated in squeezing and dripping regimes and quantitatively investigate the volumetric and morphologic variation of droplets as well as droplet parameters related to the depth direction under different flow conditions. We also demonstrated the feasibility of using this technique, as a refractive index sensor, for in-line quantitative measurement of carbamide analyte concentration within the flowing droplets.

\section{Introduction}

\footnotetext{
a. School of Mechanical Engineering and Electronic Information, China University of Geosciences, Wuhan, 430074, China, email: songcl@cug.edu.cn.

b. State Key Laboratory of Engines, Tianjin University, Tianjin 300072, China

c. State Key Laboratory of Advanced Electromagnetic Engineering and Technology

School of Electrical and Electronic Engineering, Huazhong University of Science

and Technology, Wuhan, China

d. Department of Biomedical Engineering, Southern University of Science and

Technology, Shenzhen, China

e. Queensland Micro- and Nanotechnology Centre, Griffith University, 170 Kessels

Road QLD 4111, Brisbane, Australia.

Electronic Supplementary Information (ESI) available. See DOI: 10.1039/x0xx00000x
}

Over the past decades, droplet-based microfluidics has increasingly become a favourable tool for high-throughput assays in the fields of biology, chemistry, environmental monitoring ${ }^{1,2}$. The droplets have been used as independent microreactors for performing chemical and biological reactions at femtoliter to nanoliter scales, owing to their advantages of the large interfacial areas, fast mixing, reduced sample consumption, and high throughput. As microreactors, the shape and volume of the droplets are the fundamental parameters related to the spatial distribution and amount of reactants which need to be precisely controlled in antibody and drug screening ${ }^{3,4}$ as well as in material synthesis, such as the fabrication of advanced particles ${ }^{5}$ and polymeric Janus particles ${ }^{6}$. In addition, the polymerase chain reaction (PCR) and reverse transcription (RT) PCR have been demonstrated in droplet-based microfluidics ${ }^{7-9}$. The large surface-to-volume ratios of the droplets can enhance the heat-and-mass-transfer and provide precise denaturing and annealing temperature.

Therefore, the droplet manipulation techniques for efficient transfer of chemical and biological samples such as droplet injection $^{10}$, splitting ${ }^{11}$, merging ${ }^{12}$, and vibrating ${ }^{13}$, have been intensively studied and developed to control the shape, volume, and reactant concentration of the droplets. However, current instrumentations for in situ monitoring of droplet dynamics are typically facilitated with either bright-field imaging using highspeed cameras or fluorescent imaging, and both techniques are restricted to two-dimensional (2-D) observations ${ }^{14-16}$. Some available technologies can be employed for the threedimensional (3-D) monitoring of droplets, such as confocal imaging ${ }^{17,} 18$ and optical coherence tomography ${ }^{19}$. However, these methods are usually limited to the inspection of stationary droplets, as mechanical scanning is required for image acquisition.

Recently, light-sheet fluorescent microscopy has been demonstrated $^{20}$ for 3-D imaging of flowing droplets in microchannels. However, the droplet image reconstruction of this technique depends on the distribution of fluorescent particles inside the droplet, and thus has a limited spatial 
resolution. More recently, we have proposed the use of optoacousto-fluidic microscopy for 3-D visualization of droplets with high spatial and temporal resolution 21, 22. The 3-D visualization of droplets can be re-constructed with optoacoustic signals retrieved by light-beam scanning along the transverse direction and fluidic scanning along the flowing direction. Although the lateral resolution of this technique can be enhanced to reach optical diffraction limit, the axial resolution depends on the bandwidth of ultrasound, typically on the order of $10 \mu \mathrm{m}$, which can hardly be used to resolve small morphologic features of droplets along the depth direction.

Quantitative phase imaging (QPI) has been developed as a label-free technique to investigate samples at microscale via recording sample-induced phase variation with a typical interferometric and microscopic imaging configuration ${ }^{23}$, which can be further dedicated for quantitative 3-D visualization of samples with sub-micron resolution along the axial direction ${ }^{24}$. The phase microscopy working at its transmission mode has been proposed and demonstrated for analysis of samples in microfluidic channels via inspection of sample-induced interferometric fringes, such as cells counting ${ }^{25}$ and identification ${ }^{26}$. These reported methods can only provide qualitative analysis based on sample-induced fringe pattern in the absence of phase shifting for retrieving quantitative phase information. Generally, the implementation of phase shifting requires the sample to keep at a stationary state during the process, which cannot be directly used for the measurement of microfluidic dynamics, especially when working at high throughput. In our previous work, we proposed an integrated optofluidic phase-shifting element and a differential $(5,1)$ phase-shifting algorithm to achieve quantitative measurements of micro-mixing dynamics in microchannels using a miniaturized digital holographic microscopy configuration ${ }^{27}$.

In this paper, we propose and demonstrate real-time quantitative phase microscopy (R-QPM) that can be used for 3 $D$ visualization of flowing droplets in microchannels and analysis of their volumetric, morphologic and chemical parameters via cooperatively employing the previously proposed optofluidic phase-shifting scheme ${ }^{27}$ and a high-speed linear array chargecoupled device (LACCD). Incorporating the optofluidic phaseshifting method, this imaging modality can quantitatively evaluate the phase variation induced by the flowing droplets in the microchannel. By unwrapping the phase map, the proposed imaging modality can be either used for real-time 3-D reconstruction of the droplets, or for full-field measurement of analyte concentration distribution in the droplets. In particular, we experimentally investigate the 3-D morphology of droplets formed at about $10 \mathrm{~Hz}$ with a T-junction microchannel in the squeezing and dripping regimes. Based on the 3-D visualization, droplet parameters, especially related to the depth direction, have been experimentally investigated. We also demonstrate the feasibility of using this technique, as a refractive index sensor, for the quantitative measurement of carbamide analyte concentration within the flowing droplet.

\section{Materials and method}

\section{Preparation of optofluidic chip}

The optofluidic device was fabricated using PDMS and standard soft-lithography with a channel width of $200 \mu \mathrm{m}$ and a height of $40 \mu \mathrm{m}$. The device (Fig.1 (b)) consists of two parts, one with a Tjunction microchannel (Fig.1 (c)) to produce high-throughput droplet trains, and the other with a micro-mixer to produce droplets of aqueous solution at tuneable concentration and a phase-shifting chamber (Fig.1 (e)). Silicone oil (dynamic viscosity: 0.048Pa.s, Refractive index (RI): 1.409) and aqueous analyte serving as continuous and dispersed phases respectively were pumped into the chip to form droplets at the T-junction. As to the phase-shifting element, $0.3 \mathrm{~mol} / \mathrm{L}$ calcium chloride solution and DI water were firstly fed to the micro-mixer to hydrodynamically tune the refractive index of the mixed fluid. After the RI manipulation, the mixed fluid was transported to the phase-shifting chamber and served as a wavefront retarding medium. Five steps phase shifting $(0, \pi / 2, \pi, 3 \pi / 2,2 \pi)$ can be achieved by setting five flowrate ratios. In this work, we kept the flowrate of DI water at $20 \mu \mathrm{L} / \mathrm{min}$ and the flowrate of calcium chloride solution was set at $0,4,10,20$ and $40.5 \mu \mathrm{L} / \mathrm{min}$ for each phase shifting step (Fig.1 (f)). The numerical simulation of the mixing dynamics for estimation of the switching time can be referred to Fig. S5. The detailed description of the optofluidic phase-shifting method was reported in our previous work ${ }^{27}$.

\section{Experimental setup}

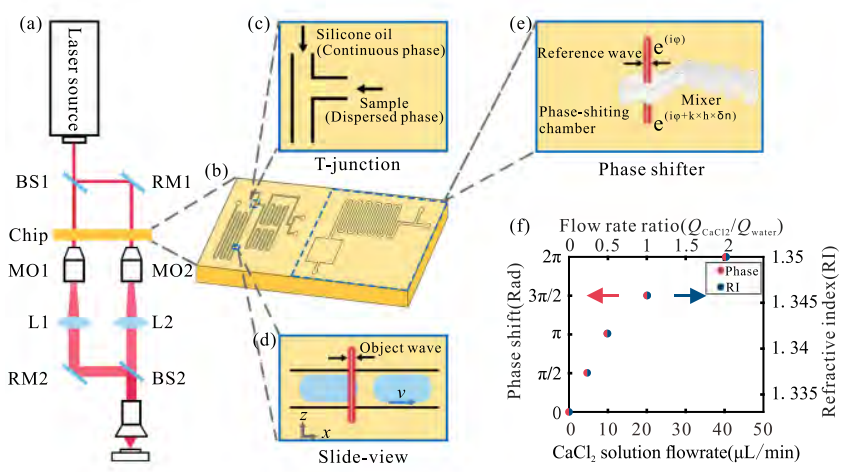

Fig. 1 (a) Schematic of the real-time quantitative phase microscopy. RM: reflector mirror, L: lens, BS: beam splitter; (b) The optofluidic chip consists of a T-junction for droplet generation on the left and a phase-shifting element on the right; (c) A T-junction for droplet generation; (d) An illustration of a droplet flowing along $x$-direction with velocity $v$ and passing through the object wave; (e) An illustration of phase-shifting principle. The shifted phase can be determined by the refractive index of the aqueous solution in the chamber that can be tuned by varying flow rates; (f) The relationship between the shifted phase ( $\mathrm{RI}$ of the aqueous solution) and the flow rate of $0.3 \mathrm{~mol} / \mathrm{L}$ calcium chloride solution when the flowrate of DI water is fixed at $20 \mu \mathrm{L} / \mathrm{min}$.

Figure 1(a) illustrates the schematic of the real-time quantitative phase microscopy (R-QPM) for the inspection of droplet-based microfluidics. A $671 \mathrm{~nm}$ laser beam launched from a single longitudinal mode laser (MSL-FN-671-100mW) was equally split into two beams by a beam splitter (BS1). One beam as the object wave was delivered to illuminate the droplets in the microchannel which flow along $x$-direction with a speed of $v$ (Fig.1 (d)), and the other beam as the reference wave was directed to the phase-shifting element (Fig. 1(e)). 
After passing through the optofluidic chip, both beams were coupled into two objectives (MO, 40X, NA=0.6), and subsequently superimposed at a beam splitter (BS2) and recorded as a linear hologram by a linear array CCD (LACCD) via an ocular. The LACCD has a resolution of $4096 \times 1$ pixels with 5$\mu \mathrm{m}$ pitch and a frame rate of $80 \mathrm{kHz}$. The total magnification of this imaging system is $48 \mathrm{X}$, and the lateral resolution along $y$ axis of the system is about $0.9 \mu \mathrm{m}$.

The resolution along the $x$-axis is related to the flow velocity of the droplets and camera frame rate. In this work, the resolution along $x$-axis is adjusted to be the same as the $y$-axis resolution by the matching the flow rate and frame rate of the camera. And the resolution along depth direction (z-axis) is estimated to be about $9 \mathrm{~nm}$. The details on the evaluation of spatial resolutions can be referred to Fig. S1 and S2. Assisted by the creeping flow in the microchannel, linear holograms can be continuously generated by the automatic fluid scanning and later stitched to reveal the information of droplet migration along the flow direction ( $x$-axis).

\section{Working principle}

The framework of real-time phase mapping is implemented by firstly recording five phase-stepping (stepping phase interval of $\pi / 2$ ) holograms as baseline holograms (Fig. 2(b)), when the channel is filled up with only the continuous phase. Each baseline hologram consists of a series of column holograms recorded by the LACCD, Fig. 2(a). The five phase-stepping holograms can be mathematically described as:

$$
I_{\mathrm{I}}=I_{\mathrm{O}}+I_{\mathrm{R}}+2 \sqrt{I_{\mathrm{O}} I_{\mathrm{R}}} \cos (\varphi+m \beta), m=0,1,2,3,4
$$

where $I_{O}$ and $I_{R}$ represent the intensities resulting from the object and the reference waves respectively, $\varphi$ is the optical phase difference between the two beams, and $\beta=\pi / 2$ denotes a constant phase stepping between each baseline holograms, which can be achieved by adjusting the refractive index of the fluid in the phase-shifting chamber, Fig. 1 (e). The relationship between the shifted phase and the refractive index is illustrated in Fig. 1(f).

After recording the five baseline holograms, fluids of both dispersed and continuous phases can be pumped into the microchannel to generate droplets at the T-junction, Fig. 1(c). When the droplets pass through the line-shaped inspection region (Fig. 1 (d)), the LACCD can be operated at filming mode to record the movement of droplets. The intensity maps of the holograms with droplet dynamics (Fig. 2(c)) can be described as:

$$
I_{2}=I_{\mathrm{O}}+I_{\mathrm{R}}+2 \sqrt{I_{\mathrm{O}} I_{\mathrm{R}}} \cos (\varphi+\Delta)
$$

where $\Delta$ is the droplet-induced phase difference.

The differential $(5,1)$ phase-shifting algorithm ${ }^{27}$ can be implemented to acquire the wrapped phase map, Fig.2 (d). Specifically, subtraction of baseline holograms from each frame of the filmed holograms can be carried out to obtain the secondary fringe patterns denoted as $I_{\mathrm{a}}, I_{\mathrm{b}}, I_{\mathrm{c}}, I_{\mathrm{d}}, I_{\mathrm{e}}$.

$$
\begin{aligned}
& I_{a, b, c, d, e}=4 \sqrt{I_{O} I_{R}}\left[\sin \left(\frac{\Delta-m \beta}{2}\right) \sin \left(\frac{\Delta+m \beta}{2}+\varphi\right)\right], \\
& m=0,1,2,3,4
\end{aligned}
$$

After filtering the high frequency component, the dropletinduced wrapped phase can be expressed as:

$$
\Delta=\arctan \left(\frac{1}{2 \sin \frac{\beta}{2}} \frac{2 I_{\mathrm{c}}-I_{\mathrm{a}}-I_{\mathrm{e}}}{I_{\mathrm{d}}-I_{\mathrm{b}}}\right)
$$

We subsequently took the PUMA algorithm for phase unwrapping to retrieve the absolute optical phase difference value $^{28}$ as shown in Fig. 2(e). The detailed phase unwrapping process was reported in our previous work ${ }^{27}$. It should be noted that the phase shifting is implemented before the droplet formation when the channel is filled up with only continuous phase fluid. The phase map can be obtained by the subtraction of each filmed hologram bearing the droplet movement from the five base-line holograms acquired during the phase-shifting process, and thus the phase map can represent the phase information at each specific point of the time-line.

The unwrapped phase map represents the optical phase difference between the state of the channel filled up with fluid of continuous phase (Fig.2(b)) and the state of the droplets passing through the object wave (Fig.2(c)). The optical phase difference can be expressed as OPD $=k \times \Delta n \times h$, where $k=2 \pi / \lambda$ is the wavenumber, $h$ denotes the droplet dimension along the $z$ direction as shown in Fig. $1(\mathrm{~b})$, and $\Delta n$ is the refractive index difference between the disperse phase and the continuous phase. Therefore, the obtained mapping of OPD ( $\Delta$ ), can be used for profiling of the droplets in 3-D given that $\Delta n$ is known as shown in Fig. 2(f). Meanwhile, the system can also be utilized as a refractive index sensor to inspect the analyte concentration in full-field provided that droplets have a constant height $h$ (Fig. $2(\mathrm{~g}))$.

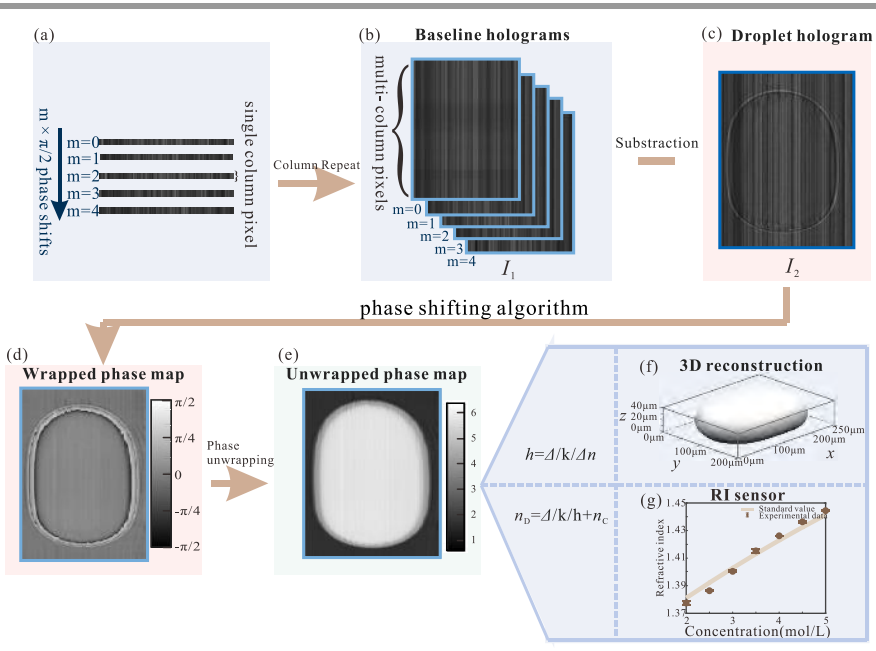

Fig. 2 (a) The five single-column holograms with a constant phase shift between each other; (b) Multi-column holograms $I_{1}$ as baseline; (c) The holograms recorded when the droplets are flowing through the inspection region; (d) The wrapped phase map obtained from the differential $(5,1)$ phase-shifting algorithm; (e) The unwrapped phase map; (f) The 3-D reconstructed visualization of a droplet provided the RI difference between the disperse and the continuous phase is known; (g) The disperse phase RI measurement under the condition of knowing the height of the droplet.

\section{Results and discussions}

Firstly, to validate the 3-D visualization of flowing droplets in real-time using the proposed R-QPM, investigations were carried out to inspect the droplets generated in squeezing and dripping regimes, and to explore the size and shape variations 
of droplets based on their 3-D imaging reconstructions. In the first experiment, $2.5 \mathrm{~mol} / \mathrm{L}$ calcium chloride solution ( $\mathrm{RI}=1.392)$ and silicone oil worked as the dispersed phase and continuous phase respectively to produce droplets at a T-junction (Fig. 1 (c)), and the total flow rate of the two phases was fixed at $240 \mu \mathrm{L} / \mathrm{h}$, which resulted in a droplet generation rate of about $10 \mathrm{~Hz}$. Prior to the generation of droplets, five baseline holograms were recorded with a $\pi / 2$ phase shift between each of them, when the inspection region was filled with silicone oil only. After switching on the generation of droplets, the LACCD can be operated at the filming mode to record sequential frames of holograms bearing the movement of the flowing droplets. The subtraction of these holograms with the five baseline holograms can result in wrapped phase map of the droplets that can be further unwrapped to reveal the quantitative droplet-induced phase variations for 3-D imaging reconstruction.

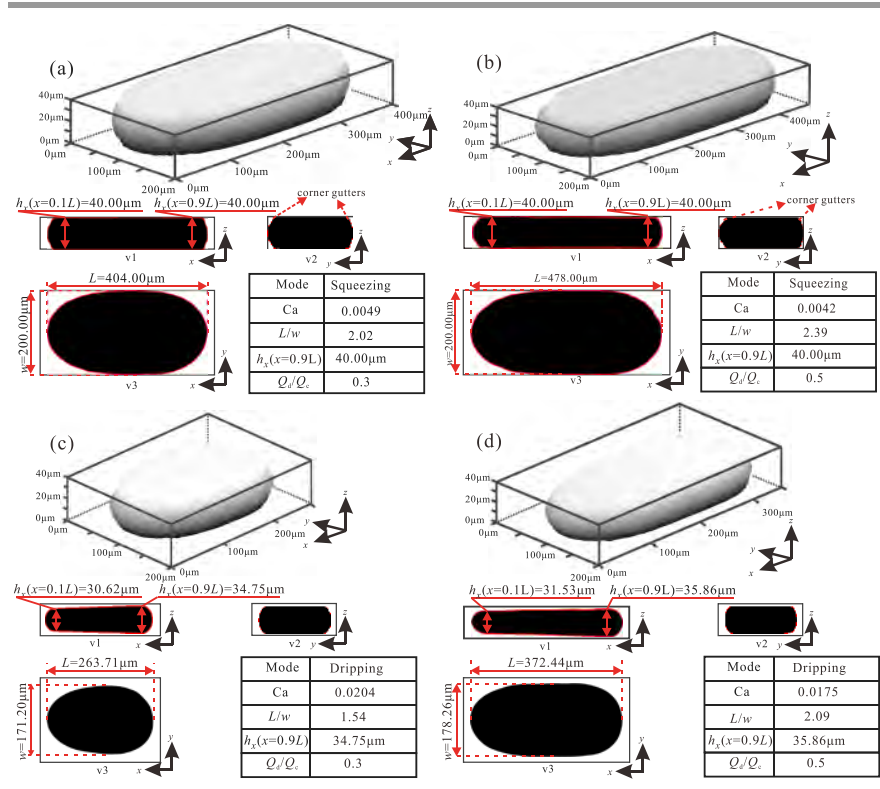

Fig. 3 The 3-D visualization and 2-D projections of droplets. (a) Droplet generated in the squeezing regime under the condition of $Q_{\mathrm{d}} / Q_{\mathrm{c}}=0.3$ and $\mathrm{Ca}=0.0049$; (b) Droplet generated in the squeezing regime under the condition of o $Q_{d} / Q_{c}=0.5$ and $\mathrm{Ca}=0.0042$. (c) Droplet generated in the dripping regime under the condition of $Q_{d} / Q_{c}=0.3$ and $\mathrm{Ca}=0.0204$.; (d) droplet generated in the dripping regime under the condition of $Q_{\mathrm{d}} / Q_{\mathrm{c}}=$ 0.5 and $\mathrm{Ca}=0.0175$.

Previous studies showed that the droplets breakup dynamics is dependent on the dimensionless capillary number $(\mathrm{Ca}=\eta v / \gamma)^{14}$, where $\eta$ is the dynamic viscosity of the continuous phase, $v$ is the velocity of the continuous phase and $v$ is the interfacial tension between the two phases. In this experiment, surfactant ( $2 \%$ mass ratio of Tween 20 ) was added into the dispersed phase to switch the droplet generation regimes from squeezing to dripping. Figures $3(a)$ and (b) illustrate the $3-D$ visualizations of droplets formed with capillary numbers of 0.0049 and 0.0042 , and flow rate ratios of 0.3 and 0.5 respectively. The droplet formation lies in the squeezing regime, as the capillary numbers are much smaller than the critical capillary number $\left(\mathrm{Ca}<O\left(10^{-2}\right)\right) .{ }^{14,}{ }^{15}$ In this case, the protrusion of the dispersed phase fluid will block the junction area, which thereby can form a pressure gradient in the continuous phase across the enlarging droplet. When the pressure gradient in the upstream is sufficiently large to overcome the pressure inside the dispersed droplet, the interface can be squeezed to deform and necked to form a droplet $^{14}$. Therefore, the droplet generated in this regime can nearly fill up the microchannels in the $y$ and $z$ directions (illustrated in the insets of side views v1 and v2 in Fig. 3 (a) and Fig. 3 (b)), and thus presents a plug shape. It was also observed that the droplets did not completely fill the rectangular microchannels but leave out the corner gutters, which show good agreement with previous studies ${ }^{29}$. Figures 3 (c) and (d) depict the 3-D visualizations of droplets formed with capillary numbers of 0.0204 and 0.0175 , and flow rate ratios of 0.3 and 0.5 respectively. The capillary number was increased by adding surfactant ( $2 \%$ mass ratio of Tween 20 ) into the calcium chloride solution. As the capillary numbers are higher than the critical capillary number $\left(\mathrm{Ca}>\mathrm{O}\left(10^{-2}\right)\right)$, the droplet formation lies in the dripping regime ${ }^{14,30}$. In this regime, the viscosity-induced shear force can drag the interface to break up before the droplets grow up to fully occupy the cross-section of the microchannel ${ }^{14}$, which results in bullet-shaped droplets (top-view in Fig. 3(c)-v3 and (d)-v3) with dimensions of $y$ and $z$ direction much smaller than those of squeezed droplets (as illustrated in Fig. 3(c) and (d)). It was observed that the droplets formed in these two cases presented wedge-shapes from the side-views shown in Figs. 3(c)-v1 and (d)-v1. These experimental results show good agreement with the previous theoretical predictions ${ }^{14,31,32}$.

As "gutter", referred to as the space between the droplet interface and the channel walls, can be used as transportation tunnel for fluid mixing ${ }^{33}$ or for synthesis of nanoparticles ${ }^{34}$ in a continuous phase slug. Thus it is of great interest to characterize the geometries of gutter for the understanding droplet flowing and heat-and-mass transfer dynamics ${ }^{35}, 36$. A great deal of efforts has been dedicated to theoretically or numerically calculate the dimensions of the gutter and its effects ${ }^{37}$. However, few experimental techniques have been reported to effectively and quantitatively inspect the gutter in 3-D. As our proposed method can resolve the flowing droplet in 3-D, especially along the height direction, it is facile to evaluate the true cross-section area of the gutter. Droplets generated in squeezing and dripping regimes were both investigated. In the experiment, $2.5 \mathrm{~mol} / \mathrm{L}$ calcium chloride solution and silicone oil worked as the dispersed phase and continuous phase respectively to produce droplets at a T-junction. Droplets of various sizes were generated by adjusting the flow rate ratio of the two phases $Q_{\mathrm{d}} / Q_{\mathrm{c}}$ from 0.05 to 0.5 , while keeping the total flow rate of the two phases at $240 \mu \mathrm{L} / \mathrm{h}$. Fig.4(a) illustrated the relationship between the dimensionless number $h_{x}(x=0.5 L) / \mathrm{H}$ and the flow rate ratio, where $h_{x}\left(I_{x}=0.5 L\right)$ and $H$ denote the thickness of the oil film $h_{\mathrm{x}}$ (shown in the insets in Fig.4(a)) and the height of the microchannel, respectively. Experimental results show that $h_{x}\left(I_{x}=0.5 L\right)$ of the droplets generated in the squeezing regime is not sensitive to the flow rate ratio, and the values of $h_{x} / \mathrm{H}$ were less than 0.08 of the channel height, which indicates that the droplets can almost fill up the channel along the height direction. However, in the dripping regime, the $h_{x} / \mathrm{H}$ 
of the droplets can be varied with the flow rate ratio from 0.18 to approximately 0.10 .

As the proposed technique can be used to reveal the thickness of oil film along the height direction, it is possible to evaluate the cross-section area of the entire gutter. Fig. 4(b) shows that the ratio $\left(R_{\mathrm{s}}=S_{\text {gutter }} / S_{\text {channel }}\right)$ between the crosssection areas of the gutter and the microchannel varies along the droplet length direction $\left(I_{x} / L\right)$, by taking the droplet in Fig. 3 (c) as an example for analysis. The re-constructed visualizations of the cross-sections of the droplet (at locations of $I_{x}=0.1 L, 0.5 L$ and $0.9 \mathrm{~L}$ ) are shown in the insets of Fig. 4 (b).

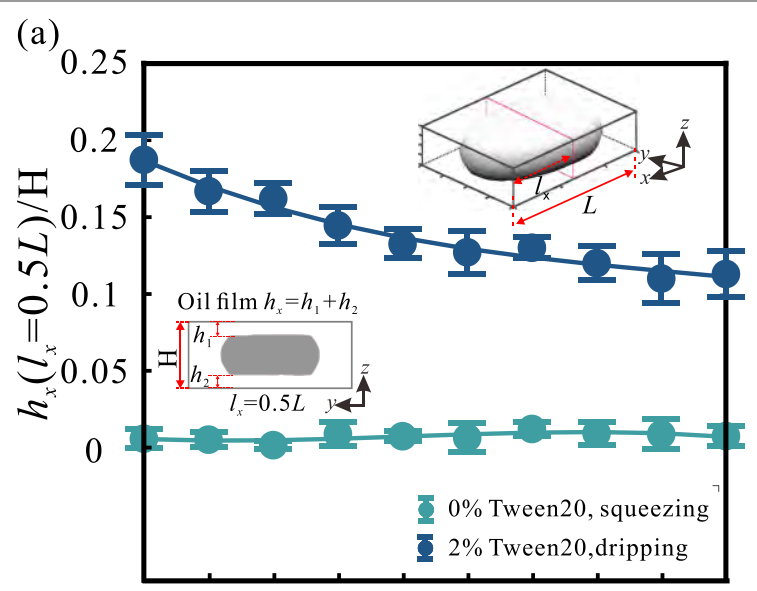

$\begin{array}{lllllllllll}0.05 & 0.1 & 0.15 & 0.2 & 0.25 & 0.3 & 0.35 & 0.4 & 0.45 & 0.5\end{array}$

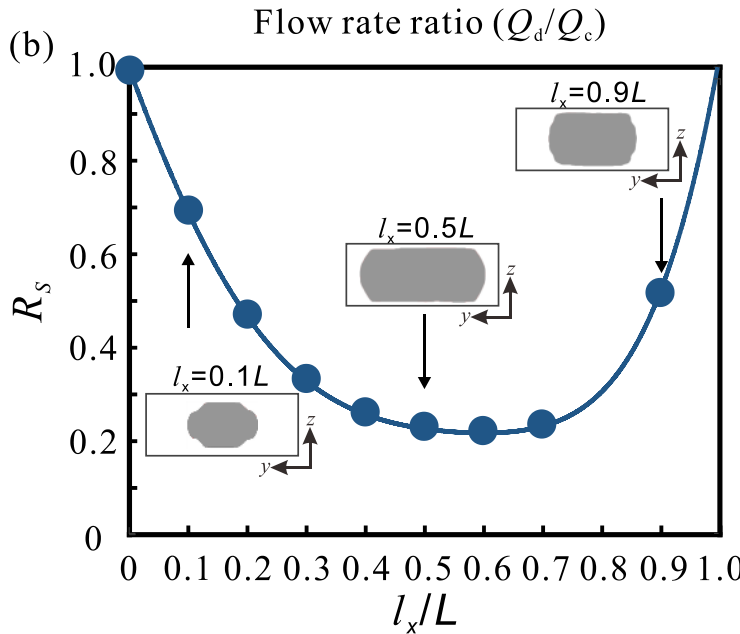

Fig. 4 (a) The relationships between the thickness of the oil film $\left(h_{x}\left(I_{x}=0.5 L\right) / H\right)$ and flow rate ratio in the squeezing (cyan data points) and dripping (blue data points) regimes; (b) The variation of cross-section area ratio $\left(R_{S}\right)$ along the droplet length direction $\left(I_{x} / L\right)$;

The Laplace pressure introduced from the curved interface of droplet can play a significant role on the motion of droplet in microfluidic network ${ }^{38,39}$. A number of applications has been developed based on the manipulation of Laplace pressure, such as droplet fusion, division and sorting by laser-heating induced pressure change ${ }^{40}$, droplet splitting and steering based on interface deformation actuated by acoustic wave ${ }^{41}$ and picoinjection of droplet via pressure fluctuation induced by the variation of channel geometries ${ }^{42}$. To date, a method for the real-time and 3-D measurement of the Laplace pressure remains a challenge. Basically, the Laplace pressure of a specific point on the droplet interface is related to the local curvature. As the proposed R-QPM can provide the morphology of the flowing droplet in 3-D, it is possible to utilise this technique to quantitatively evaluate the local curvatures of droplet interface in both $x-y$ plane and $x-z$ plane, and therefore to study the dynamics of droplet Laplace pressure in 3-D and real-time.

(a)

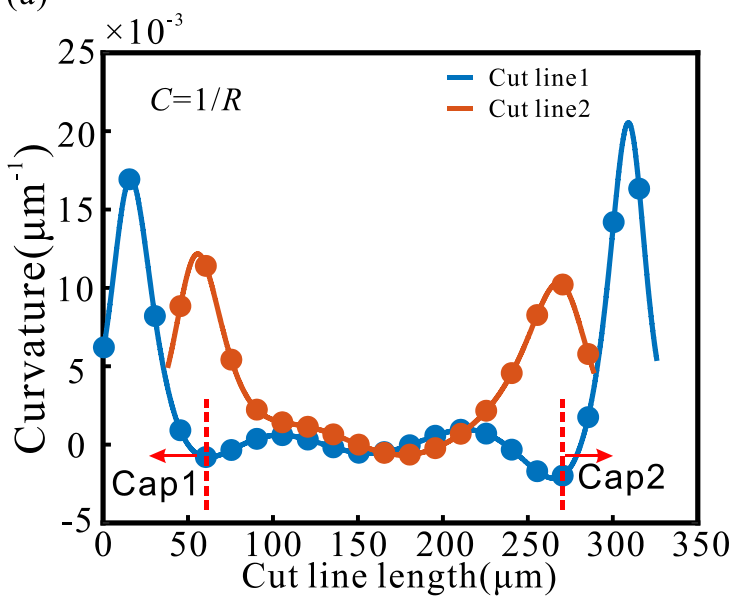

(b)

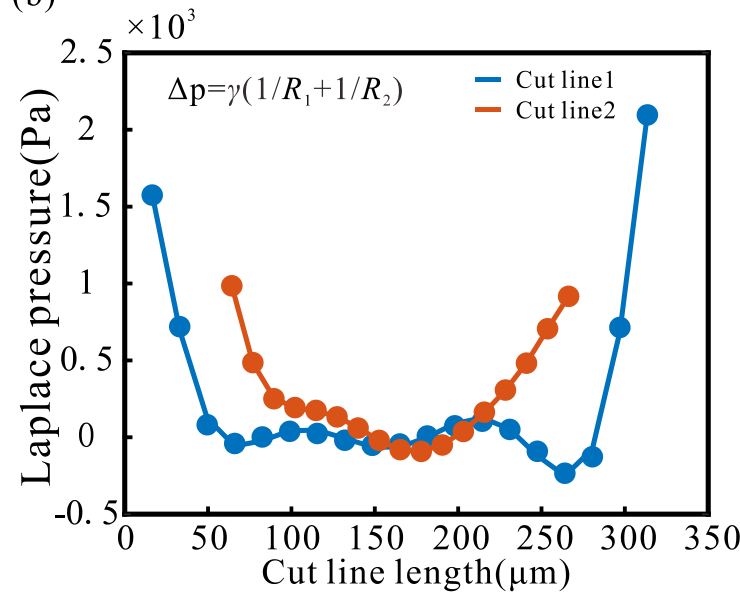

(c)

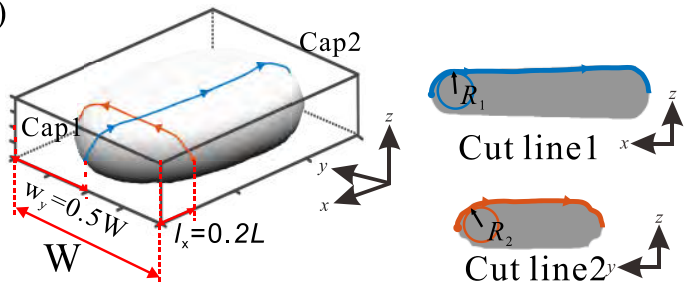

Fig. 5 (a) The curvature variation along the cut lines; (b)The Laplace pressure variation along the cut lines; (c) Illustration of the cut lines on the droplet interface for investigation.

In this analysis, we took into account the droplet that was generated, in the dripping regime, with a flow rate ratio between the two phases $Q_{\mathrm{d}} / Q_{\mathrm{c}}$ of 0.3 and the total flow rate of $240 \mu \mathrm{L} / \mathrm{h}$. Figure $5(\mathrm{a})$ shows the curvature $(C)$ variation on the droplet interface along the cut line 1 and cut line 2 . The cut line 1 is along the upper half interface of $x-z$ plane at $w_{y}=0.5 W$, where $W$ is the width of the microchannel, and the cut line 2 is along the upper half interface of $y-z$ plane at $I_{x}=0.2 L$ as shown in Fig. 5(c). The analysis shows that the local curvature varies 
across the caps of the droplet, which previously has been roughly estimated as a constant value by taking the reciprocal of the half of the height of the microchannel ${ }^{41,42}$. Based on the evaluation of the local curvature, the Laplace pressure $\Delta \mathrm{P}=\gamma\left(1 / R_{1}+1 / R_{2}\right)$, where $\gamma$ is the interface tension, $R_{1}$ and $R_{2}$ are the two radii of curvature of the interface along $x-z$ plane and $y$ $z$ plane, can be calculated and shown in Fig. 5(b). This quantitative inspection of local Laplace pressure of droplet could provide an insight on the fundamental physics for the exploration of droplet manipulation techniques.

In addition, we have also investigated the droplet surfaceto-volume ratio using the proposed R-QPM system. The surfaceto-volume ratio is an important factor, especially at the microscale, for the rate of heat and mass transfer, which can be used to precisely manipulate the chemical reaction rate in many droplet-based applications such as $\mathrm{PCR}^{7,8}$ and drug delivery ${ }^{43}$. In this study, the volumes and surface areas of the droplets under different flow conditions can be obtained by numerical integration based on the 3-D re-construction of the droplets. Fig. 6 shows the relationship between the surface-to-volume ratio and the flow rate ratio, which indicates that the droplets generated in the dripping regime generally have larger surfaceto-volume ratios comparing to the droplets generated in the squeezing regime. And in both cases, it is found that the surfaceto-volume ratio decreases with the flow rate ratio. In particular, the surface-to-volume ratio of the droplet in the dripping regime decreases more drastically than that of the droplet in the squeezed regime. The variations of volume and surface area of the droplets under different flow conditions can be referred to as Figure S3 (a) and (b), respectively.

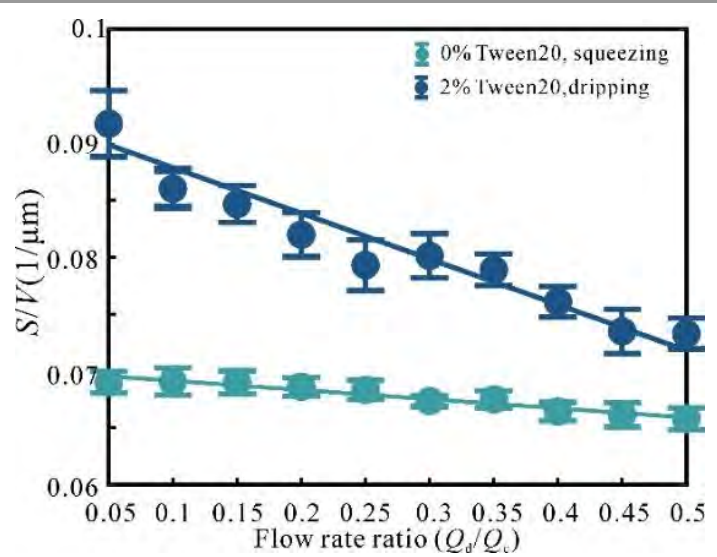

Fig. 6 The relationships between the surface-to-volume ratios and the flow rate ratios. The droplets formed in the dripping regime are presented in blue points and the cyan points represent the droplets formed in the squeezing regime.

To verify the feasibility of the proposed R-QPM for quantitative measurement of refractive index in droplet-based microfluidics, carbamide aqueous solutions were used as the dispersed phase, and silicone oil was used as the continuous phase to generate droplets in squeezing regime. Prior to the droplet formation, the saturated aqueous solutions and DI water were mixed via a micromixer as illustrated in the insets in Fig. 7, by which solutions with different concentrations can be produced by adjusting the flow rate ratio between the two inlets of the micromixer. The concentration of the well-mixed aqueous solutions can be theoretically calculated as $C=C_{0} \times Q_{1} /$ $\left(Q_{1}+Q_{2}\right)$, where $C_{0}$ is the molecular concentration of the saturated solution. The silicone oil with a refractive index of 1.409 served as the continuous phase was pumped into the optofluidic chip with flowrate $Q_{3}$, and the total flow rate $Q_{1}+Q_{2}+Q_{3}$ was kept at $240 \mu \mathrm{L} / \mathrm{h}$. After implementing the proposed method, the unwrapped phase $(\Delta)$ maps of droplets can be obtained. As the droplets were generated in the squeezing regime, the height of the droplets can be assumed to be approximately the height of the channel $(h=40 \mu \mathrm{m})$, and thus the RI mapping can be calculated using the equation $n_{D}=\Delta / k / h+n_{C}$ as described in Fig. 2. Previous studies have shown that the RI and concentration of these aqueous solutions have linear relationships ${ }^{44}, 45$. Therefore, the concentration distributions of carbamide solution in the droplets can be visualized (shown in Fig. 7 P1-P3) and quantitatively measured in Fig. 7 via extraction of optical phases using our proposed method. The experimental data show a good linear relationship with the theoretical calculations, which has a correlation coefficient of $R^{2}=0.965$.

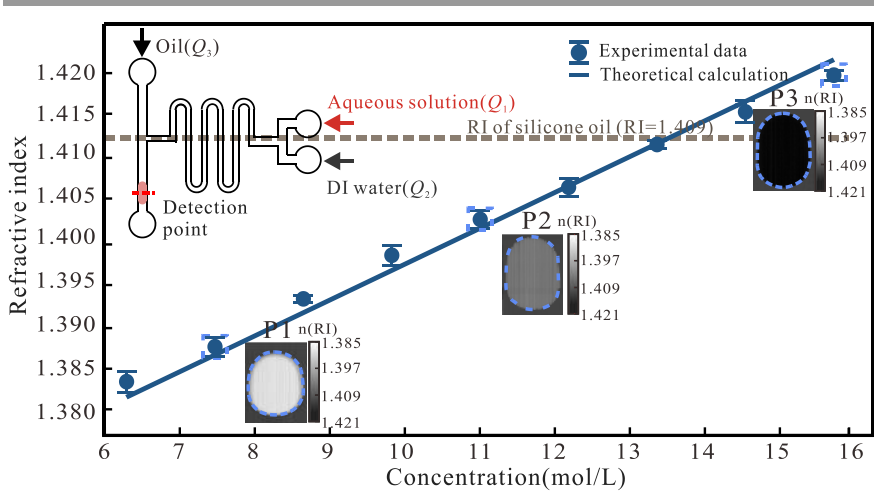

Fig.7 Quantitative measurement of refractive index of carbamide solution in dropletbased microfluidics. The measurement is carried out with droplets generated in the squeezing regime that have a constant droplet height. The quantitative evaluations refractive index of molecular concentrations can be achieved based on the visualized sample-induced phase variation (illustrated as insets P1-P3).

\section{Conclusions}

In this work, we propose and demonstrate that the real-time quantitative phase microscopy (R-QPM) can be used for on-chip three-dimensional label-free detection of droplets and highthroughput quantitative molecular measurement via real-time monitoring and extraction of sample-induced phase variation. The quantitative phase extraction can be achieved using an integrated on-chip optofluidic phase-shifting element and our recently proposed differential $(5,1)$ phase-shifting algorithm. This microscopic system shows the capability of full-field visualization of droplets, which can be used to quantitatively study the shape and size of droplets in 3-D. Experiments were carried out to investigate the droplets formed in both dripping and squeezing regimes under different flow conditions. The real-time 3-D visualization of droplets shows that droplets formed in the squeezing regime have thicknesses approximately equal to the height of the microchannel 
regardless of the flow rate ratio, while the droplets formed in the dripping regime have bullet-shaped heads from both topview and side-view, and their thicknesses are generally smaller than the height of the channel, which, to the best of the authors' knowledge, has never been experimentally observed and investigated before. Based on the 3-D visualization of flowing droplets, physical parameters such as gutter area, interface curvature, local Laplace pressure and surface-tovolume ratio of droplet, can be investigated, which can provide more insights into droplet dynamics. In addition, the R-QPM system can also be used for high-throughput quantitative measurement of molecular concentration in droplet-based microfluidics. Experiments were carried out to investigate the droplets generated in the squeezing regime using carbamide solutions. Assuming these droplets have a constant height (optical path), the extraction of sample-induced phase variation can be used to reveal the RI distributions within the droplets as well as their molecular concentrations. These demonstrated capabilities of the proposed system can hold a promise for future applications, such as study of morphologic dynamics of droplets merging, splitting and injecting or mass transport dynamics in digital PCR, chemical synthesis, and drug screening.

\section{Author contribution}

Experiments design: C. Song, Y. Luo; data acquisition: Y. Luo, J. Yang; sample preparation: $X$. Zheng, J. Wang; data analysis: Y. Luo, $X$. Tu; manuscript drafting: $Y$. Luo, C. Song; critical revision: Z. Che, J. Fang, L. Xi, N.-T. Nguyen.

\section{Conflicts of interest}

There are no conflicts to declare.

\section{Acknowledgements}

We gratefully acknowledge the financial supports from National Natural Science Foundation of China (61804138, 61905224, 61775028, 81571722) and Wuhan Science and Technology Bureau (2020010601012164).

\section{Notes and references}

1 N. Shembekar, C. Chaipan, R. Utharala and C. A. Merten, Lab on a Chip, 2016, 16, 1314-1331.

2 A. B. Theberge, F. Courtois, Y. Schaerli, M. Fischlechner, C. Abell, F. Hollfelder and W. T. Huck, Angew Chem Int Ed Engl, 2010, 49, 5846-5868.

3 L. Mazutis, J. Gilbert, W. L. Ung, D. A. Weitz, A. D. Griffiths and J. A. Heyman, Nature Protocols, 2013, 8, 870-891.

4 N. Shembekar, C. Chaipan, R. Utharala and C. A. Merten, Lab Chip, 2016, 16, 1314-1331.

5 J.-T. Wang, J. Wang and J.-J. Han, Small, 2011, 7, 1728-1754.

6 S. Lone and I. W. Cheong, RSC AdV., 2014, 4, 13322-13333.

7 W. Zhang, N. Li, D. Koga, Y. Zhang, H. Zeng, H. Nakajima, J. M. Lin and K. Uchiyama, Anal Chem, 2018, 90, 5329-5334.

8 C. D. Ahrberg, A. Manz and B. G. Chung, Lab Chip, 2016, 16, 3866-3884.
9 E. K. W. N. Reginald Beer, Lorenna Lee-Houghton, Nicholas Watkins, Shanavaz Nasarabadi, Nicole Hebert,Patrick Leung, I Don W. Arnold, | Christopher G. Bailey, and Bill W. Colston, Anal. Chem, 2008, 80, 1854-1858.

10 S. R. Doonan and R. C. Bailey, Anal Chem, 2017, 89, 40914099.

11 K. Akamatsu, K. Minezaki, M. Yamada, M. Seki and S. I. Nakao, Langmuir, 2017, 33, 14087-14092.

12 X. Niu, S. Gulati, J. B. Edel and A. J. deMello, Lab Chip, 2008, 8, 1837-1841.

13 P. Zhu, X. Tang and L. Wang, Microfluidics and Nanofluidics, 2016, 20.

14 P. Zhu and L. Wang, Lab Chip, 2016, 17, 34-75.

15 V. van Steijn, C. R. Kleijn and M. T. Kreutzer, Lab Chip, 2010, 10, 2513-2518.

16 J. Husny and J. J. Cooper-White, Journal of Non-Newtonian Fluid Mechanics, 2006, 137, 121-136.

17 Y. Yin, L. Guo, K. Chen, Z. Guo, H. Chao, B. Wang and M. Li, Sci Rep, 2018, 8, 6560.

18 Y. M. Lin, C. Song and G. C. Rutledge, ACS Appl Mater Interfaces, 2019, 11, 17001-17008.

19 S. Manukyan, H. M. Sauer, I. V. Roisman, K. A. Baldwin, D. J. Fairhurst, H. Liang, J. Venzmer and C. Tropea, J Colloid Interface Sci, 2013, 395, 287-293.

20 H. Jiang, T. Zhu, H. Zhang, J. Nie, Z. Guan, C.-M. Ho, S. Liu and P. Fei, Lab on a Chip, 2017, 17, 2193-2197.

21 C. Song, T. Jin, R. Yan, W. Qi, T. Huang, H. Ding, S. H. Tan, N. T. Nguyen and L. Xi, Lab Chip, 2018, 18, 1292-1297.

22 F. Liu, T. Jin, R. Yan, T. Li, B. Hu, L. Yao, T. Huang, C. Song and L. Xi, Opt Express, 2019, 27, 1425-1432.

23 Y. Park, C. Depeursinge and G. Popescu, Nature Photonics, 2018, 12, 578-589.

24 B. K. a. G. v. Bally, APPLIED OPTICS, 2007, 47.

25 B. Mandracchia, V. Bianco, Z. Wang, M. Mugnano, A. Bramanti, M. Paturzo and P. Ferraro, Lab Chip, 2017, 17, 2831-2838.

26 M. Ugele, M. Weniger, M. Leidenberger, Y. Huang, M. Bassler, O. Friedrich, B. Kappes, O. Hayden and L. Richter, Lab Chip, 2018, 18, 1704-1712.

27 Y. D. Luo, J. W. Yang, X. Tu, T. Y. Huang, Z. Z. Che and C. L. Song, Journal of Applied Physics, 2020, 127.

28 J. M. Bioucas-Dias and G. Valadao, IEEE Trans Image Process, 2007, 16, 698-709.

29 C. N. Baroud, F. Gallaire and R. Dangla, Lab Chip, 2010, 10, 2032-2045.

30 M. De Menech, P. Garstecki, F. Jousse and H. A. Stone, journal of fluid mechanics, 2008, 595, 141-161.

31 M. Nekouei and S. A. Vanapalli, Physics of Fluids, 2017, 29.

32 G. F. Christopher, N. N. Noharuddin, J. A. Taylor and S. L. Anna, Phys Rev E Stat Nonlin Soft Matter Phys, 2008, 78, 036317.

33 A. Gunther, S. A. Khan, M. Thalmann, F. Trachsel and K. F. Jensen, Lab Chip, 2004, 4, 278-286.

34 V. S. Cabeza, S. Kuhn, A. A. Kulkarni and K. F. Jensen, Langmuir, 2012, 28, 7007-7013.

35 M. J. Fuerstman, A. Lai, M. E. Thurlow, S. S. Shevkoplyas, H. A. Stone and G. M. Whitesides, Lab Chip, 2007, 7, 1479-1489.

36 P. Aussillous and D. Quéré, Physics of Fluids, 2000, 12.

37 Z. Che, T. N. Wong, N.-T. Nguyen and C. Yang, International Journal of Heat and Mass Transfer, 2015, 86, 455-464.

38 D. A. Sessoms, M. Belloul, W. Engl, M. Roche, L. Courbin and P. Panizza, Phys Rev E Stat Nonlin Soft Matter Phys, 2009, 80, 016317.

39 M. Prakash and N. Gershenfeld, Science, 2007, 315, 832-835.

40 C. N. Baroud, M. R. de Saint Vincent and J. P. Delville, Lab Chip, 2007, 7, 1029-1033.

41 41. J. Park, J. H. Jung, K. Park, G. Destgeer, H. Ahmed, R. Ahmad and H. J. Sung, Lab Chip, 2018, 18, 422-432. 
42 42. M. Rhee, Y. K. Light, S. Yilmaz, P. D. Adams, D. Saxena, R. J. Meagher and A. K. Singh, Lab Chip, 2014, 14, 4533-4539.

43 43. D. Liu, H. Zhang, F. Fontana, J. T. Hirvonen and H. A. Santos, Lab Chip, 2017, 17, 1856-1883.

44 44. J. R. Warren and J. A. Gordon, The Journal of Physical Chemistry, 1966, 70, 297-300.

45 C.-Y. Tan and Y.-X. Huang, Journal of Chemical \& Engineering Data, 2015, 60, 2827-2833. 